\title{
Methods of Computing Thrust Vector Coordinates for Aircrafts Equipped With Thrust Vector Control
}

\author{
Alexey $V$. Kanyshev ${ }^{1}$, Oleg N. Korsun ${ }^{2}$ and Alexander V. Stulovskii ${ }^{2, *}$ \\ ${ }^{1}$ Valery Chkalov State Flight Testing Center, Akhtubinsk, Russia \\ ${ }^{2}$ FGUP "GosNIIAS”, Moscow, Russia
}

\begin{abstract}
The paper proposes the formulas for simulation the forces and torques generated by thrust vectoring. The formulas are drawn using the polar coordinate system as well as the Rodrigues' vector rotation formula. The proposed results are confirmed by application to the processing of the aircraft flight test data.
\end{abstract}

\section{Introduction}

Nowadays thrust vectored aircrafts play an important role in aviation. Therefore, the precise mathematical models for simulation are necessary. The paper deals with the problem of simulation the forces and torques of the aircraft generated by the rotation of the thrust vector. The paper presents mathematical formulas for threedimensional projections of forces and torques drawn through the evident geometrical considerations.

The most part of publications concerning this problem consider the approaches of numerical aerodynamics, which are not suitable for solving the dynamics of flight problems [1-3]. This paper proposes the relatively simple formulas, which meet the requirements of real-time simulation of the aircraft motion. The similar approach has been used in [4] to increase the accuracy of simulating the orientation angles of the aircraft.

Let us notice that in this paper we consider the thrust as a geometric sum of vectors of input and output impulses. The paper focuses on calculating the forces and torques created by the rotation of output impulse vector because the simulation of the effect of input impulse is similar for thrust vectoring aircrafts and other types of the aircraft.

\section{The model of forces and torques generated by the thrust vector rotation}

The methods for describing a direction toward a point in three-dimensional space are well known. Let us choose the polar coordinate system shown in figure 1 . In this case $\varphi$ is an angle between vector and horizontal plane, whereas $\psi$ is an angle between projection of vector on horizontal plane and a coordinate axis. Using this method in regards to aircraft thrust vector we can determine thrust projections on aircraft principal axes as:

$$
\begin{gathered}
P_{x}=P \cos \varphi \cos \psi ; \\
P_{y}=P \sin \varphi ; \\
P_{z}=P \cos \varphi \sin \psi .
\end{gathered}
$$

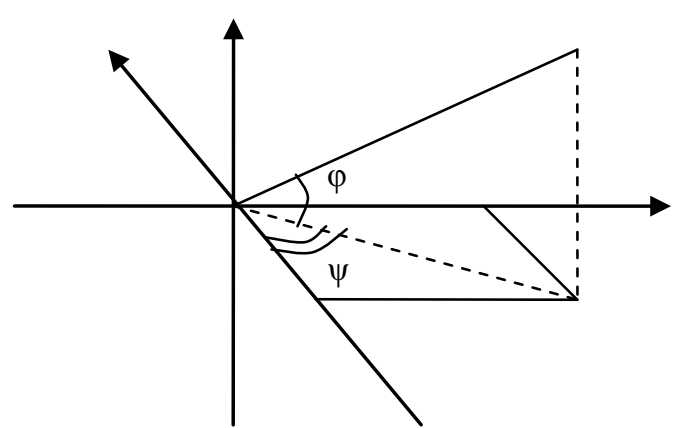

Fig. 1. Example of defining the direction in three-dimensional space by two orientation angles.

This simple model enables to describe engine thrust vector rotation assuming that nozzles are capable of turning in vertical and horizontal planes independently.

In practice, engine thrust nozzles are often capable of rotating only in single plane. This plane may be positioned at some angle to vertical plane, enabling generation of moments about all three axes simultaneously. Let us draw the mathematic expressions for torques and forces.

\subsection{Vector rotating in a plane}

Let us assume that plane of thrust vector rotation is positioned at known angle $\chi$ relative to vertical plane. At the same time $\eta$ is the angle of rotation in this plane. The zero value of this angle $\eta=0$ corresponds to vector lying in horizontal plane. It is also assumed that thrust vector crosses the center of nozzle's section. Let us draw axis parallel to aircraft's longitudinal axis, which comes through the center of nozzle's section in its zero position.

Corresponding author: avstlv2@,gmail.com 
Let us choose two points $\mathrm{A}$ and $\mathrm{O}$ on this axis. Point $\mathrm{O}$ is a point of nozzle rotation, point $\mathrm{A}$ is positioned in the center of nozzle's section. Next, draw vertical plane, which includes line AO. Let us also draw rotation plane, which includes line $\mathrm{AO}$ and is positioned at angle $\chi$ to vertical plane (figure 2).

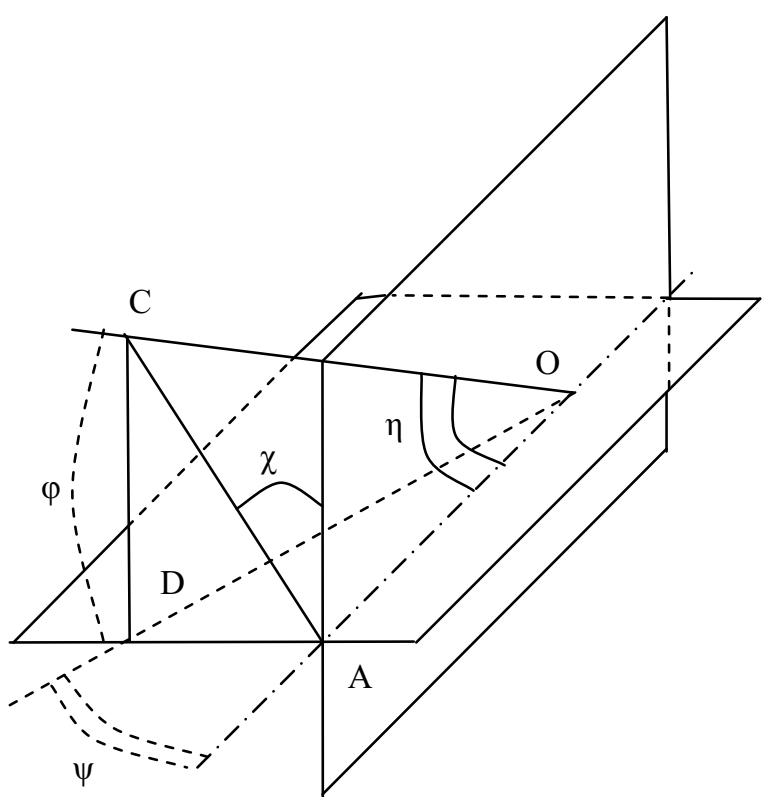

Fig. 2. Scheme connecting couples of angles $\chi, \eta$ и $\psi, \varphi$.

Let us assume that nozzle is turned by an angle $\eta$. Then the thrust vector would pass through the point $\mathrm{C}$. From the point $\mathrm{C}$ let us draw perpendicular $\mathrm{CD}$ to horizontal plane.

Let us determine relations between couples of angles $\psi, \varphi$ and $\eta, \chi$. First, we would derive expression for angle $\psi$. Since the triangles AOD and AOC are the right triangles, the next expressions are true:

$$
\begin{aligned}
& A C=A O t g \eta, \\
& A D=A O t g \psi .
\end{aligned}
$$

Triangle $\mathrm{ADC}$ is also a right triangle, therefore

$$
\sin \chi=A D / A C \text {. }
$$

The expression for the angle $\psi$ could be derived from the following equation

$$
\operatorname{tg} \psi=\operatorname{tg} \eta \sin \chi
$$

Second, we derive expression for the angle $\varphi$. Since the triangles $\mathrm{CDO}$ and $\mathrm{AOC}$ are the right triangles, the next formulas are true:

$$
\begin{aligned}
& A C=O C \sin \eta, \\
& C D=O C \sin \varphi .
\end{aligned}
$$

The triangle $\mathrm{ADC}$ is also the right triangle, therefore $\cos \chi=C D / A C$.

Value of $\varphi$ can be obtained from following equation:

$$
\sin \varphi=\sin \eta \cos \chi
$$

Finally, the formulas for values of angles $\psi, \varphi$ in terms of angles $\chi, \eta$ are

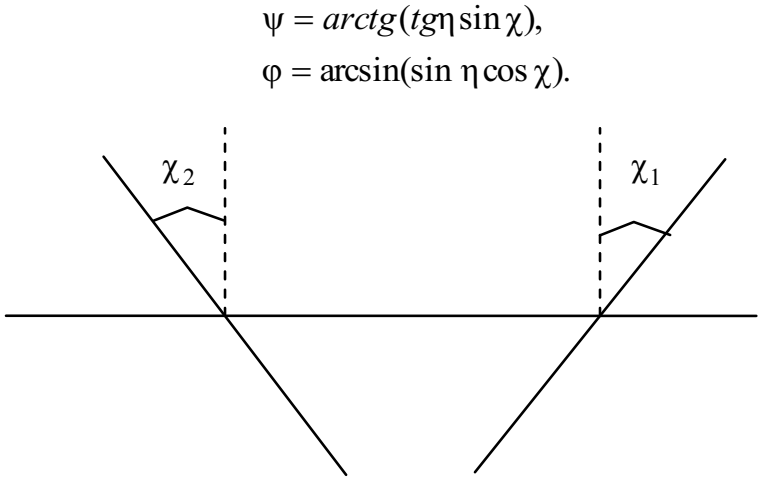

Fig. 3. Schematic picture of engine nozzle rotation planes viewed from the aircraft's tail.

Let us further consider projections of engine thrust vector on aircraft principal axes. Let us determine the signs for nozzles deviations in the same way as the signs of deviations of other control surfaces of the aircraft.

According to this rule, angle $\chi$ is positive for right engine $\left(\chi_{1}>0\right)$ and negative for left engine $\left(\chi_{2}>0\right)$ (figure 3). Angle $\eta$ is positive when nozzle goes down and negative when nozzle goes up.

Let us assume that both nozzles are turned upwards. According to above formulas for angles $\psi, \varphi$ we can easily determine the signs of thrust projections. Actually, in this case angle $\varphi$ is negative for both engines while $\psi$ is negative for right engine and positive for left one. Therefore, both engines will generate positive projections of engine thrust on longitudinal axis and negative projections on normal axis. Projections on lateral axis will have different signs, "+" for left engine and "-" for right engine.

Then let us assume that both nozzles are turned downward. In this case angle $\varphi$ is positive for both engines, and angle $\psi$ is positive for right engine and negative for left one. Both engines generate positive projections of engine thrust on longitudinal and normal axes. Right engine generates positive projection and left engine generates negative projection on lateral axis.

Results described above correspond to engineering common sense, and confirm correctness of proposed formulas.

\subsection{Arbitrary rotating vector}

Above mentioned way of computing thrust vector projections requires nozzles to be parallel to aircraft's longitudinal axis in their initial state. In case of arbitrary initial direction of thrust vector first method is inapplicable. Therefore, we should consider other methods for description of rotation. One such method is known as Rodrigues' rotation formula describing rotation of vector about arbitrary unit vector, when both vectors have common initial point.

Let $\mathbf{v}$ be vector of engine thrust in nozzle's initial state. Let $\mathbf{e}$ be a unit vector about which $\mathbf{v}$ rotates and $\eta$ - angle of rotation. Coordinates of these vectors 
should be given in body axis coordinate system. Then Rodrigues' formula is:

$$
\mathbf{v}_{\mathbf{r o t}}=(\mathbf{e} \cdot \mathbf{v}) \mathbf{e}+|\mathbf{e} \times \mathbf{v}|\left(\mathbf{e}_{1} \cos \eta+\mathbf{e}_{2} \sin \eta\right),
$$

where $\mathbf{v}_{\text {rot }}$ is result of vector $\mathbf{v}$ rotation, $\mathbf{e}_{\mathbf{1}}$ and $\mathbf{e}_{\mathbf{2}}$ with $\mathbf{e}=\mathbf{e}_{3}$ constitute right-handed basis and are defined by formulas

$$
\begin{gathered}
\mathbf{e}_{1}=\frac{\mathbf{e} \times(\mathbf{v} \times \mathbf{e})}{|\mathbf{e} \times \mathbf{v}|}, \\
\mathbf{e}_{2}=\frac{\mathbf{e} \times \mathbf{v}}{|\mathbf{e} \times \mathbf{v}|} .
\end{gathered}
$$

In Rodrigues' formula first expression corresponds to part of vector $\mathbf{v}$ collinear with $\mathbf{e}$, whereas second is orthogonal to it. Opening the brackets, we obtain

$$
\mathbf{v}_{\text {rot }}=(\mathbf{e} \cdot \mathbf{v}) \mathbf{e}+\mathbf{e} \times(\mathbf{v} \times \mathbf{e}) \cos \eta+\mathbf{e} \times \mathbf{v} \sin \eta .
$$

After using vector triple product formula

$$
\mathbf{a} \times(\mathbf{b} \times \mathbf{c})=\mathbf{b}(\mathbf{a} \cdot \mathbf{c})-\mathbf{c}(\mathbf{a} \cdot \mathbf{b}),
$$

we derive formula describing rotated vector:

$$
\mathbf{v}_{\text {rot }}=(\cos \eta) \mathbf{v}+(\sin \eta) \mathbf{e} \times \mathbf{v}+(1-\cos \eta)(\mathbf{e} \cdot \mathbf{v}) \mathbf{e}
$$

We can obtain projections of thrust vector by multiplying rotated vector by value of thrust:

$$
\mathbf{P}=\left(\begin{array}{c}
P_{x} \\
P_{y} \\
P_{z}
\end{array}\right)=|\mathbf{P}| \cdot \mathbf{v}_{\text {rot }}
$$

Component-wise this formula can be written:

$$
\begin{aligned}
& P_{x}=|\mathbf{P}|\left(v_{x} \cos \eta+\left(e_{y} v_{z}-e_{z} v_{y}\right) \sin \eta+(1-\cos \eta)(\mathbf{e} \cdot \mathbf{v}) e_{x}\right), \\
& P_{y}=|\mathbf{P}|\left(v_{y} \cos \eta+\left(e_{z} v_{x}-e_{x} v_{z}\right) \sin \eta+(1-\cos \eta)(\mathbf{e} \cdot \mathbf{v}) e_{y}\right), \\
& P_{z}=|\mathbf{P}|\left(v_{z} \cos \eta+\left(e_{x} v_{y}-e_{y} v_{x}\right) \sin \eta+(1-\cos \eta)(\mathbf{e} \cdot \mathbf{v}) e_{z}\right) .
\end{aligned}
$$

The next step is to count torque using classical expression:

$$
\mathbf{M}=\mathbf{r} \times \mathbf{P} .
$$

Component-wise torque can be written as:

$$
\begin{aligned}
M_{x} & =y\left(P_{z 1}+P_{z 2}\right)-z_{1} P_{y 1}-z_{2} P_{y 2} ; \\
M_{y} & =z_{1} P_{x 1}+z_{2} P_{x 2}-x\left(P_{z 1}+P_{z 2}\right) ; \\
M_{z} & =x\left(P_{y 1}+P_{y 2}\right)-y\left(P_{x 1}+P_{x 2}\right) .
\end{aligned}
$$

\section{Example of proposed model application}

As an example of applications of a presented model let us consider the problem of aircraft parameter identification. The accuracy of the identification is usually associated with the condition number of Fisher information matrix. This number in its turn depends on correlation between the flight signals involved into identification process. So the introduction of additional parameters - angles of nozzle deviation can easily upset this balance because of the strong correlation between nozzle deviations and other control surfaces' signals.

Let us consider a set aircraft flight data obtained through flight test experiment. Let us calculate the torques generated by the engine nozzle's rotation using the above formulas and the value of thrust determined through the engine gas-dynamic model. Further, let us assume these torques as known functions and subtract them out of the flight signals. Thus, we exclude the influence of thrust vectoring. It enables to use the traditional identification methods [5] to obtain aerodynamic coefficient's estimates

As an example let us determine the efficiency coefficients of aircraft control surfaces in lateral channel and estimate the control torque produced by flaperons. Let us also compare these estimates obtained from flight data with corresponding torques calculated through known bank of this aircraft aerodynamic parameters. Results of this comparison in flaperon channel are shown in figure 4. In this case, happily, we obtain high degree of coincidence between two independent sources, that is the parameter flight data identification and the bank of aerodynamic parameters, drawn from wind tunnel tests. It confirms the validity of proposed engine thrust rotation model.

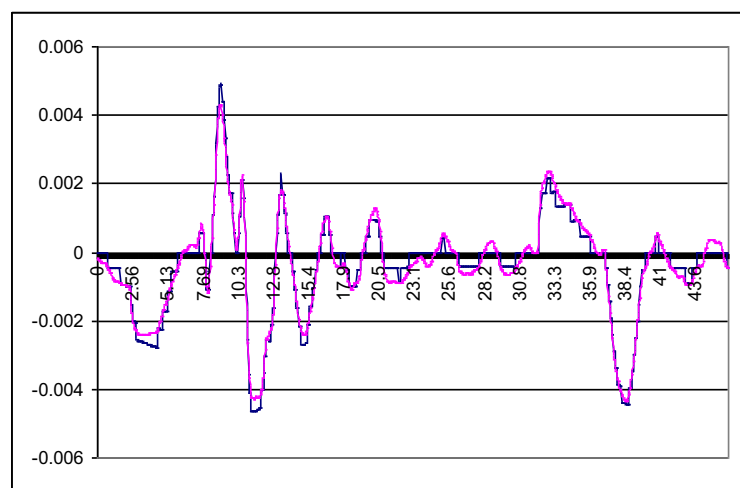

Fig. 4. Values of torque about normal axis generated by flaperons obtained from coefficients bank (blue line) and through parameter identification (violet line).

\section{Conclusions}

In this paper the algorithms to compute the forces and torques produced by engine thrust vector rotation are proposed. The paper also presents an example of testing this model validity using the results of aircraft parameter identification based on the flight data.

\section{References}

1. B. Gel-Or, Journal of thermal science, 7, 1-6 (1998)

2. R. Deng, F. Kong, H.D. Kim, Journal of mechanical science and technology, 28, 4979-4987 (2014)

3. L. Li, M. Hirota, K. Ouchi et al., Shock waves, 27, 53-61 (2016)

4. Bulgakov, V.V., Korsun, O.N., Kulabukhov, V.S. et al., Journal of computer and systems sciences international, 55, 150 (2016)

5. O.N. Korsun, B.K. Poplavsky, 29th Congress of the International Council of the Aeronautical Sciences, ICAS 2014; St. Petersburg, 2014-0210. 